\title{
Large prevertebral hematoma and carotid pseudoaneurysm following percutaneous anterior cervical discectomy: illustrative case
}

\author{
Gregory W. Basil, MD, Annelise C. Sprau, BS, Robert M. Starke, MD, Allan D. Levi, MD, and Michael Y. Wang, MD \\ Department of Neurological Surgery, University of Miami, Miami, Florida
}

\begin{abstract}
BACKGROUND The percutaneous, endoscope-assisted anterior cervical discectomy is a relatively new procedure, and because of its novelty, complications are minimal and pertinent literature is scarce. This approach relies on a sufficient anatomical understanding of the vital neurovascular structures in the operating workspace. Although complications are rare, they can be significant.

OBSERVATIONS The patient presented with difficulty breathing following an anterior percutaneous cervical discectomy performed at an outpatient surgical center. Imaging revealed a prevertebral hematoma and multiple carotid pseudoaneurysms. Given the large prevertebral hematoma and concern for imminent airway collapse, the authors proceeded with emergent intubation and surgical evacuation of the clot.

LESSONS The authors propose managing complications in a fashion similar to those for comparable injuries after classic anterior approaches. Definitive management of our patient's carotid injury would require stenting and, therefore, dual antiplatelet agents. Thus, the authors proceeded with the hematoma evacuation first. Additionally, careful dissection was needed to decrease further carotid damage. Thus, the authors made a more rostral incision to maintain the given stability of the carotid insult before the angiographic intervention to follow. It is the authors' hope that the technical pearls from this two-staged open hematoma evacuation and endovascular stenting may guide future presurgical and intraoperative planning and management of complications, should they arise.
\end{abstract}

https://thejns.org/doi/abs/10.3171/CASE2055

KEYWORDS carotid injury; management; percutaneous anterior cervical approach; prevertebral hematoma

The percutaneous, endoscope-assisted anterior cervical discectomy is a relatively new procedure, yet it has been well described in the literature. ${ }^{1}$ This approach relies on sufficient anatomical understanding and technical mastery to safely access the cervical disc space without injury to surrounding critical neurovascular structures. Although anterior cervical discectomies and artificial cervical disc replacements are proven minimally disruptive approaches to cervical pathologies, it has been suggested that anterior endoscopic approaches to the cervical spine may help avoid early cervical fusions. ${ }^{2}$

However, it is critical to recognize that because these approaches rely on percutaneous techniques, they do not allow for direct identification and protection of critical anatomical structures. Indeed, access to the disc space often relies on the surgeon using his or her fingers to press downwards against the spine and develop a safe corridor between the esophagus and carotid artery. ${ }^{1,3}$ Although the reported rate of complications of this procedure is low, there have been notable reports of potentially devastating complications, mostly related to access. ${ }^{4}$
These complications include carotid injury, esophageal injury, and prevertebral hematoma formation..$^{1,4,5}$

In this case lesson, we discuss the management of a patient who presented with acute-onset dysphonia and dyspnea following a percutaneous endoscope-assisted anterior cervical discectomy and was found to have a large prevertebral hematoma with an associated carotid injury. To our knowledge, this is the first report proposing a detailed management paradigm for such a complication.

\section{Illustrative Case \\ Background}

A 28-year-old female presented to our emergency department with the chief complaints of dysphonia and increasing difficulty breathing following an anterior percutaneous cervical discectomy performed approximately 10 hours earlier at an outpatient surgical center. She had been given a printout of intraoperative fluoroscopy shots from the 
surgery, which demonstrated a likely percutaneous approach to the C4-5 level with the disc space accessed at approximately midline (Fig. 1). She reported a history of left-sided C5 radiculopathy leading up to her procedure and denied any new neurological complaints at the time of her presentation.

On physical examination, the patient was noted to have a small horizontal incision on the left aspect of her neck, approximately halfway between midline and the sternocleidomastoid muscle, roughly at the level of the thyroid cartilage. There was a mild-to-moderate area of bruising extending approximately $1 \mathrm{~cm}$ circumferentially around the incision. The incision was closed with several interrupted nylon sutures, and there was notable induration without discharge. The patient's neck was diffusely firm to palpation, more prominently on the left than on the right, and tender throughout. The patient had significantly muffled speech, often typically described as a "hot potato" voice. She was saturating at $100 \%$ on a nonrebreather mask but became mildly dyspneic with speech.

As a result of these symptoms, and given concerns for a hematoma in the surgical bed and possible vascular injury, computed tomography and computed tomography angiography (CTA) scans of the neck were immediately obtained and demonstrated a large prevertebral hematoma extending all the way from the clivus to the T2 level, measuring approximately $4.1 \times 5.7 \times 15.0 \mathrm{~cm}$. CTA additionally demonstrated two irregularly-shaped, contrast-filled focal outpouchings of the distal left common carotid artery, one projecting anteriorly and measuring $5 \times 3 \times$ $3 \mathrm{~mm}$ and the other projecting posteriorly and measuring $4 \times 3 \times 4 \mathrm{~mm}$ (Fig. 2). These findings were thought to represent likely iatrogenic pseudoaneurysms, from a single through-and-through carotid injury.

\section{Management}

Given the large prevertebral hematoma and concern for imminent airway collapse, the decision was made to proceed with emergent intubation and surgical evacuation of the clot. The patient was brought to the operating room with a tracheostomy kit open. Anesthesia was planned for an awake fiberoptic versus a video-assisted intubation. Upon further airway assessment, we felt that a video-assisted intubation would be possible. Intubation was uneventful, and the patient was then positioned supine on the bed and prepped and draped in the usual sterile fashion.

The main concern during surgical planning was deciding the best way to avoid disturbing the carotid injury at the site of her previous surgery. Therefore, the decision was made to open a separate horizontal incision, also on the left and approximately $1.5 \mathrm{~cm}$ above the original incision. The incision was made using a 10-blade knife, and the platysma was immediately encountered and noted to be stained with dark red hematoma. Sharp dissection was carefully performed, and the large prevertebral hematoma was encountered and noted to be displacing the esophagus and trachea anteriorly. The hematoma capsule was incised, and a large quantity of the clot was evacuated from the prevertebral space using a combination of blunt dissection and suction. A hemovac was placed into the resulting potential space and tunneled out to the skin, lateral to the new incision. The esophagus and surrounding tissues were carefully inspected with no obvious injury noted. The patient was left intubated and brought to the intensive care unit, with the plan for endovascular stent placement the following day.

There were no issues in the immediate postoperative period, and the patient was examined and found to be grossly neurologically intact without clinical evidence of cerebrovascular insult. Treatment options for the carotid injury were discussed with the patient and family and included balloon test occlusion with carotid sacrifice versus carotid stenting. Ultimately, the family opted for the placement of a carotid stent if indicated. The next day, the patient was taken to the angiography suite where a cerebral angiogram was obtained and redemonstrated two left common carotid pseudoaneurysms without evidence of active extravasation (Fig. 3). As a result, a stent was placed in the left common carotid artery with no further filling of the pseudoaneurysms noted (Fig. 4).

Following her procedure, the patient was left intubated and started on dual antiplatelet agents along with steroid taper to reduce airway edema to prepare for extubation. She was successfully extubated on postoperative day 4 and was neurologically intact on examination. At the 3-week follow-up, she remained asymptomatic and neurologically intact, and the revision incision was well healed and approximated. Her original incision demonstrated peripheral necrosis and wound
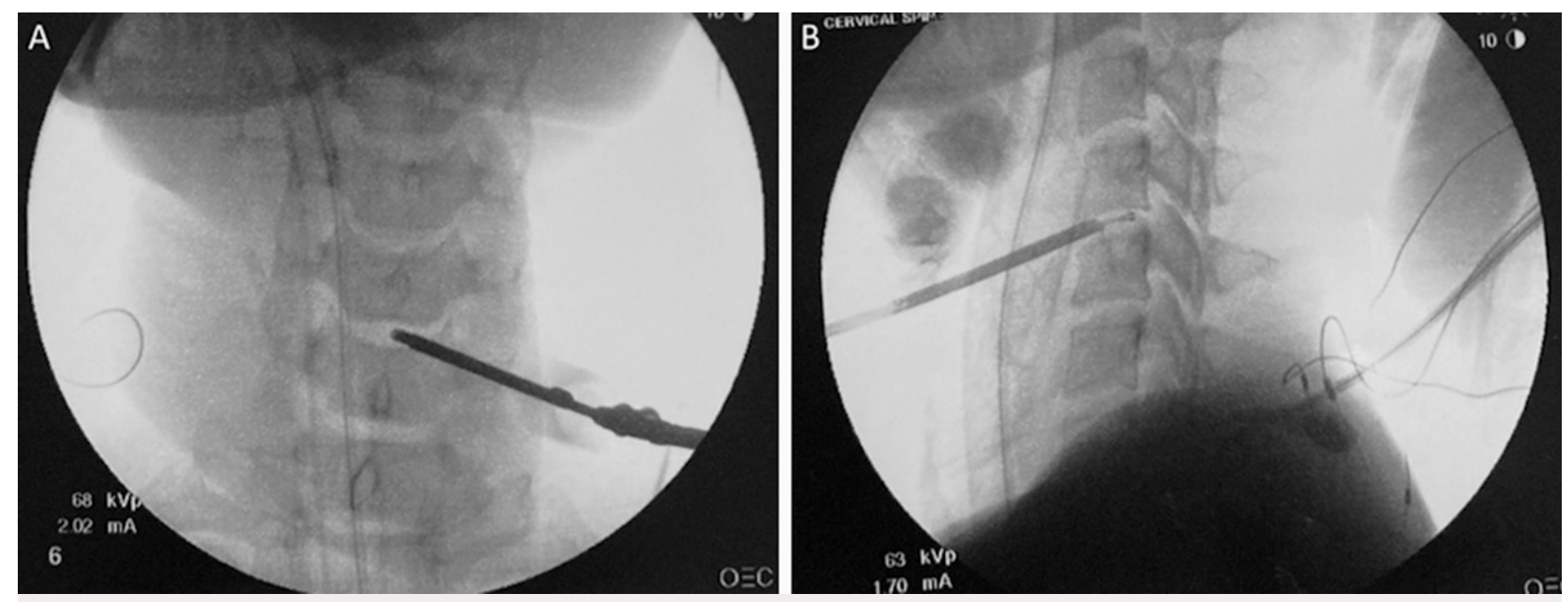

FIG. 1. Intraoperative fluoroscopy shots, anterior-posterior (A) and lateral (B) views, provided by the patient, demonstrating a percutaneous endoscopic approach to the C4-5 disc space accessed at approximately midline. 

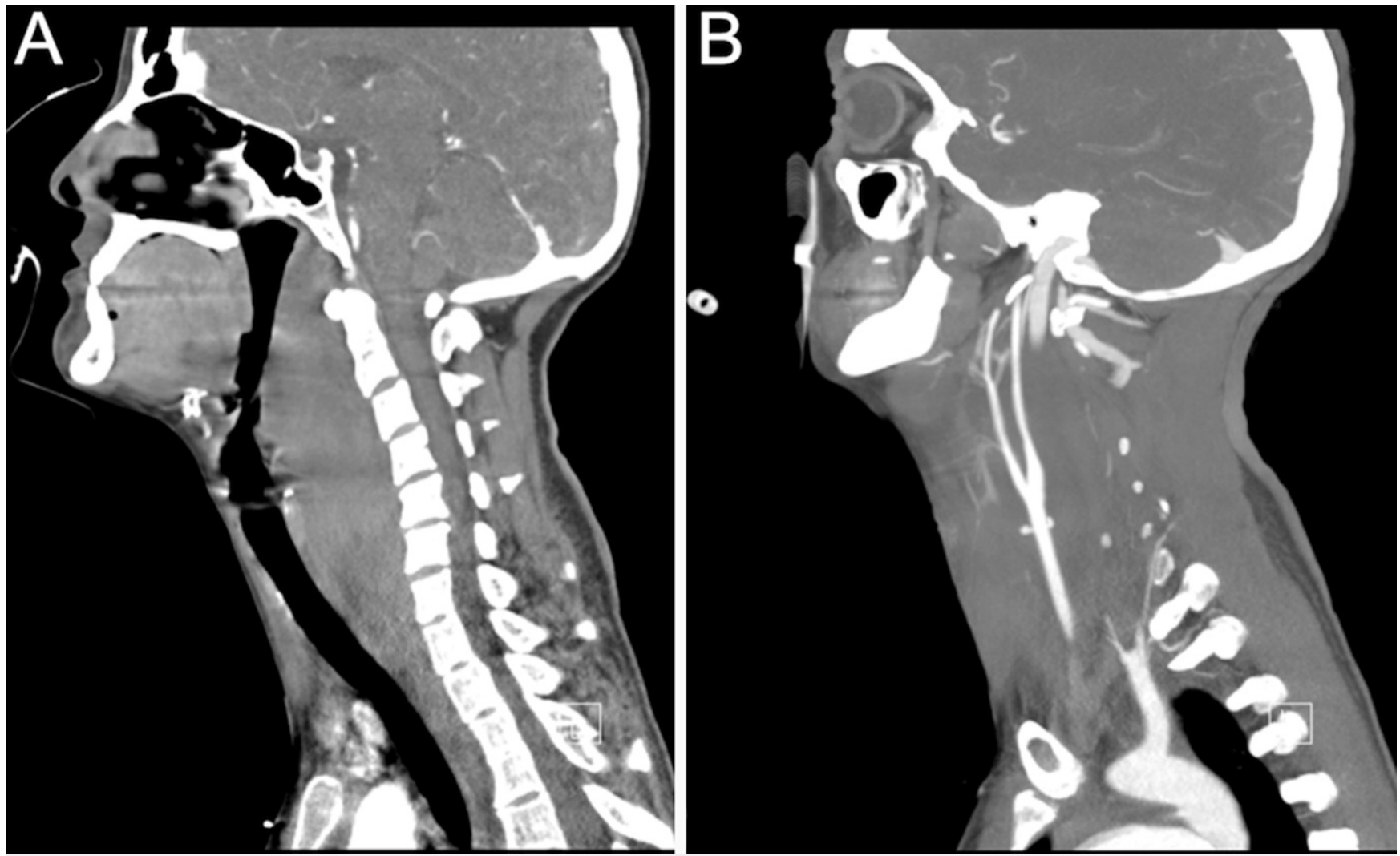

FIG. 2. CTA of the left neck demonstrating a large prevertebral hematoma extending from the clivus to the T2 level (A) and 2 outpouchings of the common carotid artery just proximal to the carotid bifurcation (B).

dehiscence (Fig. 5). The dehiscent wound was cleaned and redressed, and she was referred to plastic surgery for wound care follow-up.

\section{Discussion}

\section{Observations}

This, to our knowledge, is the first report describing the management of a large prevertebral hematoma with an associated carotid injury after percutaneous endoscope-assisted anterior cervical discectomy. Though advances in endoscopic management of cervical pathology may decrease the risk of adjacent spondylosis compared with the risks with classic open approaches, this technique relies on a robust anatomical grasp of the cervical territory to prevent potentially devastating complications. ${ }^{6}$ It is our hope that the features described in this case lesson may guide future presurgical and intraoperative planning and management of complications, should they arise.

Cervical pathology has historically been treated with anterior cervical discectomy, bone graft fusion and internal fixation, or posterior decompression and fusion. Though classic anterior approaches to the cervical spine have been exhaustively studied and validated, long-term follow-up has revealed complications due to accelerated degeneration of adjacent segments, as well as the reappearance of related symptoms and signs of cervical spondylosis. ${ }^{7,8}$ Full endoscopic approaches aim to avoid these complications by preserving the biomechanical stability of the spine by limiting tissue disruption. ${ }^{9}$ In the reported literature, indications for percutaneous anterior approaches are limited, however, and this surgery is often performed on isolated soft disc herniations causing radiculopathy. Further, Shen at al. ${ }^{10}$ highlight that this technique is primarily offered by senior surgeons given the technical capability needed to master this approach. Ultimately, endoscopy relies on operating using fluoroscopic guidance, thus increasing the potential to damage critical anatomical structures due to less visualization. ${ }^{10}$

In the execution of the percutaneous anterior approach, the carotid artery and esophagus are two vital structures that a surgeon must be cautious to avoid. However, this region also houses several large veins, including the internal jugular vein; multiple branching vessels from the carotid; and several critical nerves, including the recurrent laryngeal nerve. Therefore, although the complication rate for this approach is rather low, potential injuries can be significant. To date, the largest case series from Tzaan ${ }^{1}$ of 107 patients undergoing anterior percutaneous endoscopic approaches reported a complication rate of $2 \%$, including an iatrogenic carotid injury with resulting stroke and neurological deficit. The author briefly discusses management of the carotid injury with a stent placement. ${ }^{1}$ The most common complications reported in the literature are access related and typically appear early, including vascular injury, prevertebral hematoma, laryngeal edema, and esophageal perforation. ${ }^{3}$

The technically demanding portion of the approach lies in the structures anterior to the intravertebral disc itself. Advancing dilators through the narrow operating cannula requires a precise amount of force because resistance is normally met when encountering the anterior portion of the disc space. ${ }^{11}$ This can become particularly 


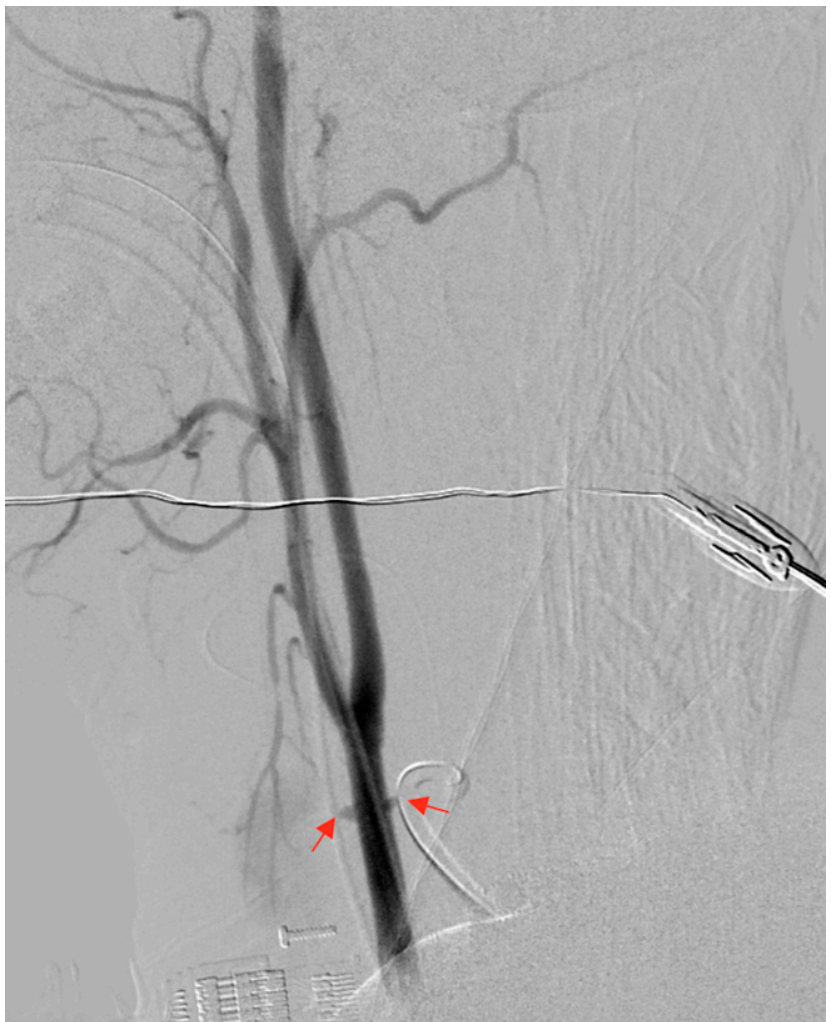

FIG. 3. Left common carotid injection demonstrating 2 pseudoaneurysms (red arrows) without evidence of active extravasation.

difficult in patients with a narrow anterior disc size because a small error in the trajectory can injure the carotid artery. Thus, it is recommended to exclude patients with disc spaces of less than $4 \mathrm{~mm}$ in the anterior height or those with bony pathology blocking the anterior aspect of the disc space. ${ }^{1}$ Lee and Gastambide report another case of carotid injury that they attribute to the patient's small, short neck. ${ }^{12}$ This further underscores the importance of careful patient selection for anatomical amenability to an endoscopic approach.

\section{Lessons}

Prevertebral hematomas and carotid injuries occur rarely but must be rapidly detected to prevent airway obstruction and other compressive injuries. ${ }^{13}$ Based on the radiographic evidence, it is our belief that the present hemorrhagic complication was not due to inadequate hemostasis but rather due to a direct carotid insult. Additionally, a large retrospective radiographic study of percutaneous intervention from Jeon et al. found that the left C5-6 levels had a significantly higher incidence of vascular structures in the prevertebral areas, thus potentially contributing to our patient's postoperative complication because her primary intervention was on the left. ${ }^{5}$

Management of such complications have not, to date, been reported in the literature, likely because of the novelty of this technique and overall complication infrequency. We do, however, argue for managing these patients in a fashion similar to those for comparable injuries after classic anterior approaches. The surrounding body of literature reports that critical cases with rapidly expanding hematomas and carotid injury, such as the present case, should be emergently

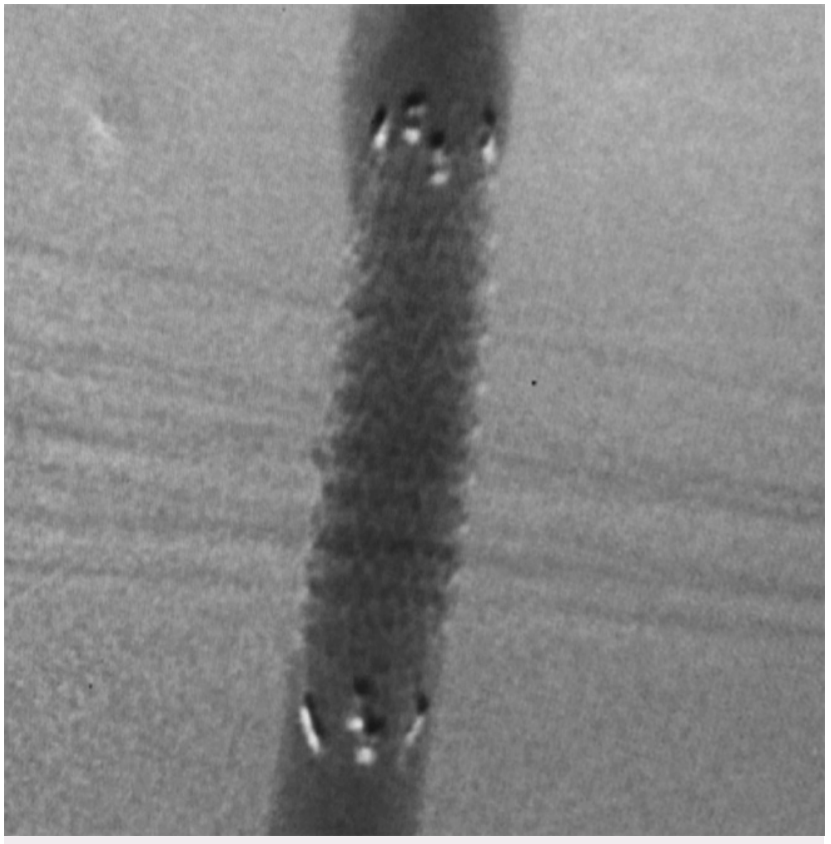

FIG. 4. Left common carotid injection following placement of the stent with no evidence of further filling of previously visualized pseudoaneurysms.

intubated to prevent respiratory compromise before surgical evacuation. ${ }^{14,15}$ It is critical that this intubation be performed with the patient awake (or, as in our case, using videoscopic guidance) and with a tracheostomy kit available to enable swift intervention should the patient lose their airway during attempted intubation. This management paradigm is well described in the literature. ${ }^{16-18}$

The next question would be whether to address the hematoma or carotid injury first. In the case of our patient, we were concerned that definitive management of her carotid injury would require stenting and, therefore, dual antiplatelet agents. This would theoretically make her hematoma evacuation and subsequent recovery somewhat more challenging. Therefore, we decided to proceed with the evacuation of the hematoma prior to treatment of the carotid injury.

Typically, the prior surgical incision is reopened to allow for hematoma visualization and evacuation. Our case was unique in that careful dissection was needed to decrease the risk of further carotid damage. Thus, we proceeded with a separate, more rostral incision to maintain the given stability of the carotid insult before the angiographic intervention to follow.

latrogenic extracranial carotid pseudoaneurysms treated with endovascular stenting have exhibited good outcomes in the adult populations, resulting in aneurysm occlusion and preservation of the artery without significant complications. ${ }^{19}$ We decided to treat the carotid pseudoaneurysms endovascularly with a stent given its safe and high technical success rate at large academic institutions. ${ }^{20}$ To prevent associated thrombosis, embolism, or vessel dissection, our patient was placed on dual antiplatelet therapy after successful endovascular intervention.

Prevertebral hematomas and carotid injuries are rare yet devastating complications following percutaneous, endoscope-assisted cervical discectomy and are rarely reported in the literature. Our two-staged open hematoma evacuation and endovascular stenting 


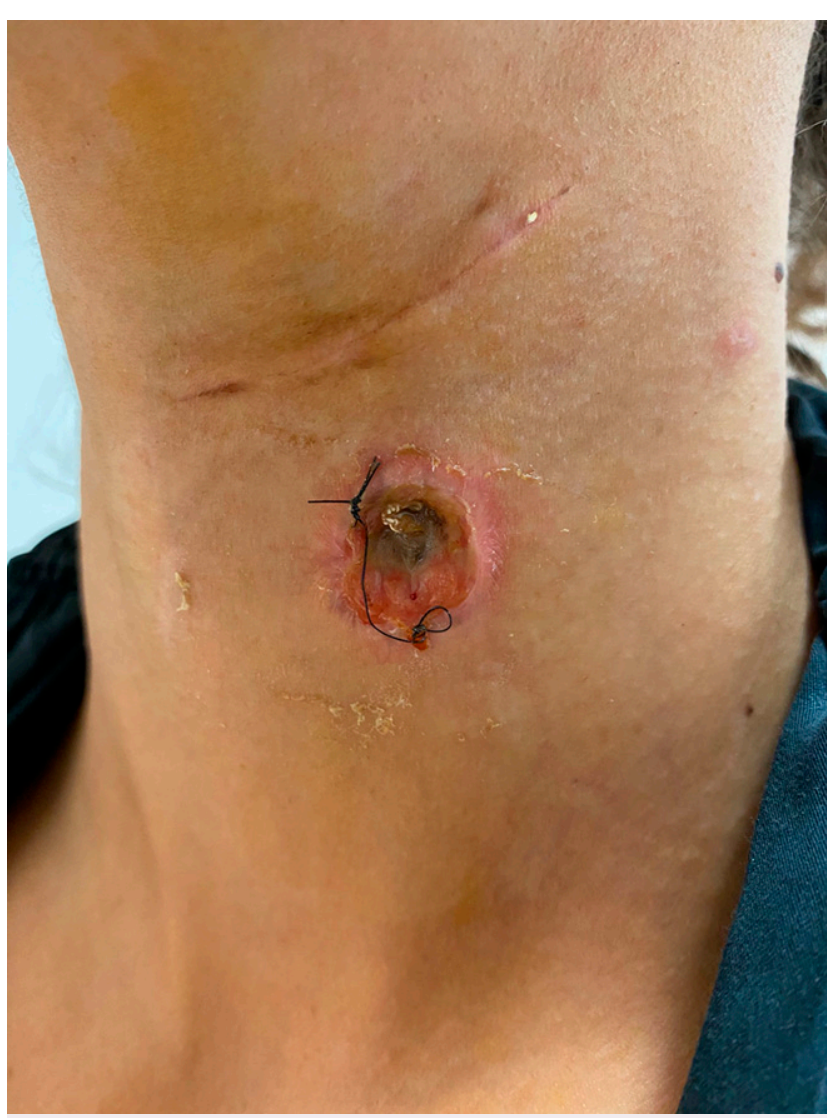

FIG. 5. Three-week postoperative image demonstrating an approximated and well-healed anterior transverse incision. Inferior circular incision from the primary surgery demonstrating wound dehiscence and peripheral necrosis with granulation tissue.

effectively decompressed the trachea and esophagus and allowed for carotid repair without vascular sacrifice. The complexity of complications presented in this case underlines the importance of patient selection for percutaneous endoscopic approaches and details the necessity of dynamic surgical intervention for resolution. Thus, we highlight pearls from our successful hematoma evacuation and carotid stabilization and offer suggestions for surgical planning for future complication avoidance.

\section{Acknowledgments}

Dr. Levi has received grants from the National Institutes of Health and the Department of Defense for work outside the submitted work.

\section{References}

1. Tzaan WC. Anterior percutaneous endoscopic cervical discectomy for cervical intervertebral disc herniation: outcome, complications, and technique. J Spinal Disord Tech. 2011;24(7): 421-431.

2. Fujibayashi S, Takemoto M, Nakamura T, et al. Stand-alone anterior cervical discectomy and fusion using an additive manufactured individualized bioactive porous titanium implant without bone graft: results of a prospective clinical trial. Asian Spine J. Published online September 22, 2020. doi:10.31616/ asj.2020.0231.
3. Quillo-Olvera J, Lin GX, Kim JS. Percutaneous endoscopic cervical discectomy: a technical review. Ann Trans/ Med. 2018;6(6): 100.

4. Oh HS, Hwang BW, Park SJ, et al. Percutaneous endoscopic cervical discectomy (PECD): an analysis of outcome, causes of reoperation. World Neurosurg. 2017;102:583-592.

5. Jeon JK, Oh $\mathrm{CH}$, Chung $\mathrm{D}$, et al. Prevertebral vascular and esophageal consideration during percutaneous cervical disc procedures. Spine (Phila Pa 1976). 2014;39(4):275-279.

6. Jiang $Z$, Wang $A$, Wang $C$, et al. Analysis of compliance and efficacy of integrated management of whole process in the choice of percutaneous full-endoscopic surgery for patients with cervical disc herniation. J Orthop Surg Res. 2020;15(1):381.

7. Chen J, Liu Z, Zhong G, et al. Surgical treatment for cervical spondylotic myelopathy in elderly patients: a retrospective study. Clin Neurol Neurosurg. 2015;132:47-51.

8. Kong W, Xin Z, Du Q, et al. Anterior percutaneous full-endoscopic transcorporeal decompression of the spinal cord for single-segment cervical spondylotic myelopathy: the technical interpretation and 2 years of clinical follow-up. J Orthop Surg Res. 2019;14(1):461.

9. Lee JH, Lee $\mathrm{SH}$. Clinical and radiographic changes after percutaneous endoscopic cervical discectomy: a long-term follow-up. Photomed Laser Surg. 2014;32(12):663-668.

10. Shen J, Telfeian AE, Shaaya E, et al. Full endoscopic cervical spine surgery. J Spine Surg. 2020;6(2):383-390.

11. Deng ZL, Chu L, Chen L, et al. Anterior transcorporeal approach of percutaneous endoscopic cervical discectomy for disc herniation at the C4-C5 levels: a technical note. Spine J. 2016;16(5):659-666.

12. Lee SH, Gastambide D. Perkutane endoskopische Diskotomie der Halswirbelsäule. In: Pfeil J, Siebert W, Janousek A, Josten C, eds. Minimal-invasive Verfahren in der Orthopädie und Traumatologie. Springer; 2000:41-61.

13. McDowell MM, Parry PV, Agarwal N, et al. Long term delay in onset of prevertebral hematoma following anterior cervical discectomy and fusion: a case report. J Clin Neurosci. 2019;62: 234-237.

14. Lee SH, Kim KT, Jeong BO, et al. The safety zone of percutaneous cervical approach: a dynamic computed tomographic study. Spine (Phila Pa 1976). 2007;32(20):E569-E574.

15. Pait TG, Killefer JA, Arnautovic KI. Surgical anatomy of the anterior cervical spine: the disc space, vertebral artery, and associated bony structures. Neurosurgery. 1996;39(4):769-776.

16. Palumbo MA, Aidlen JP, Daniels AH, et al. Airway compromise due to wound hematoma following anterior cervical spine surgery. Open Orthop J. 2012;6:108-113.

17. Song KJ, Choi BW, Lee DH, et al. Acute airway obstruction due to postoperative retropharyngeal hematoma after anterior cervical fusion: a retrospective analysis. J Orthop Surg Res. 2017;12(1): 19.

18. Kuhn JE, Graziano GP. Airway compromise as a result of retropharyngeal hematoma following cervical spine injury. J Spinal Disord. 1991;4(3):264-269.

19. Pinzón M, Lobelo NO, Rodríguez MC, et al. Endovascular management of iatrogenic cervical internal carotid artery pseudoaneurysm in a 9-year-old child: case report and literature review. Int J Pediatr Otorhinolaryngol. 2017;95:29-33.

20. Alaraj A, Wallace A, Amin-Hanjani S, et al. Endovascular implantation of covered stents in the extracranial carotid and vertebral arteries: case series and review of the literature. Surg Neurol Int. 2011;2:67.

\section{Disclosures}

Dr. Basil has direct ownership in Kinesiometrics LLC. Dr. Levi has a finanical relationship with the American Association of Neurological Surgeons outside of the work submitted. Dr. Wang receives royalties 
from DePuy Synthes; has relationships with DePuy Synthes, ISD, Medical Device Partners, Kinesiometrics, and Biocomposites outside the work submitted; and has received personal fees from Spineology, Globus Medical, and Medtronic.

\section{Author Contributions}

Conception and design: Wang, Basil, Starke, Levi. Acquisition of data: Wang, Basil, Starke, Levi. Analysis and interpretation of data: Basil, Starke, Levi. Drafting the article: all authors. Critically revising the article: Basil, Starke, Levi. Reviewed submitted version of manuscript: Basil, Sprau, Starke, Levi. Approved the final version of the manuscript on behalf of all authors: Sprau. Administrative/ technical/material support: Basil, Starke, Levi. Study supervision: Basil, Starke, Levi.

\section{Correspondence}

Michael Y. Wang: University of Miami, Miami, FL. mwang2@ med.miami.edu. 